\title{
Some results of meromorphic solutions of second-order linear differential equations
}

Junfeng $\mathrm{Xu}^{1 *}$ and Xiaobin Zhang ${ }^{2}$

\author{
${ }^{*}$ Correspondence: \\ xujunf@gmail.com \\ ${ }^{1}$ Department of Mathematics, Wuyi \\ University, Jiangmen, Guangdong \\ 529020, P.R. China \\ Full list of author information is \\ available at the end of the article
}

\begin{abstract}
In this paper, we investigate the growth and the zeros of difference of the first and second derivative of the solutions of the second-order linear differential equations

$$
f^{\prime \prime}+A_{0} e^{a_{0} z} f^{\prime}+\left(A_{1} e^{a_{1} z}+A_{2} e^{a_{2} z}\right) f=0
$$

and a small function, where $A_{j}(z)(\not \equiv 0)(j=0,1,2)$ are meromorphic functions and $a_{0}<0, a_{1} a_{2} \neq 0, a_{1} \neq a_{2}$. Our result extended the results of Peng and Chen, Belaïdi and others.

MSC: $34 \mathrm{M} 10 ; 30 \mathrm{D} 35$

Keywords: linear differential equation; meromorphic function; growth order; Nevanlinna theory; small function
\end{abstract}

\section{Introduction and main results}

In this paper, we shall assume that the reader is familiar with the fundamental results and the standard notation of the Nevanlinna value distribution theory of meromorphic functions (see [1-3]). The term 'meromorphic function' will mean meromorphic in the whole complex plane $\mathbb{C}$. In addition, we will use notations $\rho(f)$ to denote the order of growth of a meromorphic function $f(z), \lambda(f)$ to denote the exponents of convergence of the zerosequence of a meromorphic function $f(z), \bar{\lambda}(f)$ to denote the exponents of convergence of the sequence of distinct zeros of $f(z)$.

In order to give some estimates of fixed points, we recall the following definitions (see $[4,5])$.

Definition 1.1 Let $f$ and $g$ be two meromorphic functions satisfying $\rho(g)<\rho(f)$, and let $z_{1}, z_{2}, \ldots\left(\left|z_{j}\right|=r_{j}, 0 \leq r_{1} \leq r_{2} \leq \cdots\right)$ be the sequence of distinct zeros of the meromorphic function $f-g$. Then $\bar{\tau}_{g}(f)$, the exponent of convergence of the sequence of distinct zeros of $f-g$, is defined by

$$
\bar{\tau}_{g}(f)=\inf \left\{\tau>\left.0\left|\sum_{j=1}^{\infty}\right| z_{j}\right|^{-\tau}<+\infty\right\} .
$$

It is evident that

$$
\bar{\tau}_{g}(f)=\varlimsup_{r \rightarrow \infty} \frac{\log \bar{N}\left(r, \frac{1}{f-g}\right)}{\log r}
$$

and $\bar{\tau}_{g}(f)=\bar{\lambda}(f-g)$. 
Clearly, if $g(z)=z$, then Definition 1.1 is equivalent to the definition of the exponent of convergence of the sequence of distinct fixed points of $f(z)$. We denote $\bar{\tau}_{z}(f)=\bar{\tau}(f)$.

For the second-order linear differential equation

$$
f^{\prime \prime}+e^{-z} f^{\prime}+B(z) f=0
$$

where $B(z)$ is an entire function of finite order, it is well known that each solution $f$ of (1.1) is an entire function. If $f_{1}$ and $f_{2}$ are any two linearly independent solutions of (1.1), then at least one of $f_{1}, f_{2}$ must have infinite order [6]. Hence, 'most' solutions of (1.1) will have infinite order.

Thus a natural question is: What condition on $B(z)$ will guarantee that every solution $f \not \equiv 0$ of (1.1) will have infinite order? Frei, Ozawa, Amemiya and Langley, and Gundersen studied the question. For the case that $B(z)$ is a transcendental entire function, Gundersen [7] proved that if $\rho(B) \neq 1$, then for every solution $f \not \equiv 0$ of (1.1) has infinite order.

In 2002, Chen considered the problem and obtained the following result in [8].

Theorem A Let $a, b$ be nonzero complex numbers and $a \neq b, B(z) \neq 0$ be a nonconstant polynomial or $B(z)=h(z) e^{b z}$, where $h(z)$ is a nonzero polynomial. Then every solution $f$ $(\neq \equiv)$ of the equation

$$
f^{\prime \prime}+e^{b z} f^{\prime}+B(z) f=0
$$

has infinite order.

Theorem B Suppose that $A_{j}(z)(\not \equiv 0)(j=0,1)$ are entire functions and $\rho\left(A_{j}\right)<1$, let $a, b$ be complex numbers and $a b \neq 0$ and $a \neq b$, then every solution $f(\equiv 0)$ of the equation

$$
f^{\prime \prime}+A_{1} e^{a z} f^{\prime}+A_{0} e^{b z} f=0
$$

has infinite order.

Recently in [9], Peng and Chen have investigated the order and the hyper-order of solutions of some second-order linear differential equations and have proved the following result.

Theorem C Suppose that $A_{j}(z)(\not \equiv 0)(j=1,2)$ are entire functions and $\rho\left(A_{j}\right)<1$, let $a_{1}$, $a_{2}$ be complex numbers such that $a_{1} a_{2} \neq 0$, and let $a_{1} \neq a_{2}$ (suppose that $\left|a_{1}\right| \leq\left|a_{2}\right|$ ). If $\arg a_{1} \neq \pi$ or $a_{1}<-1$, then every solution $f(\not \equiv 0)$ of the equation

$$
f^{\prime \prime}+e^{-z} f^{\prime}+\left(A_{1} e^{a_{1} z}+A_{2} e^{a_{2} z}\right) f=0
$$

has infinite order and $\rho_{2}(f)=1$.

In this paper, we extend and improve the above result from entire solutions to meromorphic solutions. 
Theorem 1.1 Suppose that $A_{j}(z)(\not \equiv 0)(j=0,1,2)$ are meromorphic functions and $\rho\left(A_{j}\right)<1$, and $a_{1}, a_{2}$ are two complex numbers such that $a_{1} a_{2} \neq 0, a_{1} \neq a_{2}$ (suppose that $\left.\left|a_{1}\right| \leq\left|a_{2}\right|\right)$. Let $a_{0}$ be a strictly negative real constant. If $\arg a_{1} \neq \pi$ or $a_{1}<a_{0}$, then every solution $f(\equiv 0)$, whose poles are of uniformly bounded multiplicities, of the equation

$$
f^{\prime \prime}+A_{0} e^{a_{0} z} f^{\prime}+\left(A_{1} e^{a_{1} z}+A_{2} e^{a_{2} z}\right) f=0
$$

has infinite order and $\rho_{2}(f)=1$.

Remark 1 It has been shown by Bank [10] that the growth of a meromorphic solution of a linear differential equation with meromorphic coefficients cannot be estimated uniformly in terms of the growth of the coefficients alone. Bank [10] (see also [11]) stated his result in terms of an example. Hence, in addition to the growth of the coefficients, some additional piece of information is needed when estimating the growth of meromorphic solutions. Bank and Laine [12] have shown that the number of zeros of a solution should also be taken into account in the meromorphic coefficients case. Chiang and Hayman ([13], Corollary 2.5) showed that we can estimate the growth of a meromorphic solution, when $\delta(\infty)>0$, in terms of the Nevanlinna characteristics of the coefficients. The condition $\delta(\infty)>0$ is sharp because $\delta(\infty)=0$ in Bank's example. In this paper, we give an additional condition that the solutions whose poles are of uniformly bounded multiplicities can guarantee the growth of the poles of the meromorphic solution less than or equal to the meromorphic coefficients. We can change the condition that the multiplicity of the poles is uniformly bounded to $\delta(\infty)>0$ when we considered the hyper order by using Lemma 2.7.

Since the beginning of the last four decades, a substantial number of research articles have been written to describe the fixed points of general transcendental meromorphic functions (see [14]). In [4], Chen first studied the problems on the fixed points of solutions of second-order linear differential equations with entire coefficients. Since then, Wang and Yi [15], Laine and Rieppo [16], Chen and Shon [17], Liu and Zhang [18], El Farissi and Belaïdi [19] studied the problems on the fixed points of solutions of second-order linear differential equations with meromorphic coefficients and their derivatives.

The other main purpose of this paper is to study the exponent of convergence of the sequence of distinct fixed points of all solutions of equation (1.2). In fact, inspired by [ 5 , $20-22]$, we can generalize the fixed-point to the small function.

Theorem 1.2 Let $A_{j}(z), a_{j}$ satisfy the additional hypotheses of Theorem 1.1. If $\varphi(\not \equiv 0)$ is a meromorphic function whose order is less than 1 , then every meromorphic solution $f \not \equiv 0$, whose poles are of uniformly bounded multiplicities, of equation (1.2) satisfies

$$
\bar{\lambda}(f-\varphi)=\bar{\lambda}\left(f^{\prime}-\varphi\right)=\bar{\lambda}\left(f^{\prime \prime}-\varphi\right)=\infty
$$

Corollary 1 Let $A_{j}(z), a_{j}$ satisfy the additional hypotheses of Theorem 1.1. If $f \neq 0$ is a meromorphic solution, whose poles are of uniformly bounded multiplicities, of equation (1.2), then $f, f^{\prime}, f^{\prime \prime}$ all have infinitely fixed points and satisfy

$$
\bar{\tau}(f)=\bar{\tau}\left(f^{\prime}\right)=\bar{\tau}\left(f^{\prime \prime}\right)=\infty
$$




\section{Lemmas}

The following lemma, due to Gross [23], is important in the factorization and uniqueness theory of meromorphic functions, playing an important role in this paper as well. We give a slightly changed form as follows.

Lemma 2.1 ([22]) Suppose that $f_{1}(z), f_{2}(z), \ldots, f_{n}(z)(n \geq 2)$ are meromorphic functions and $g_{1}(z), g_{2}(z), \ldots, g_{n}(z)$ are entire functions satisfying the following conditions:

(i) $\sum_{j=1}^{n} f_{j}(z) e^{g_{j}(z)} \equiv f_{n+1}$.

(ii) If $1 \leq j \leq n+1,1 \leq k \leq n$, the order of $f_{j}$ is less than the order of $e^{g_{k}(z)}$. If $n \geq 2$,

$1 \leq j \leq n+1,1 \leq h<k \leq n$, and the order of $f_{j}(z)$ is less than the order of $e^{g_{h}-g_{k}}$.

Then $f_{j}(z) \equiv 0(j=1,2, \ldots, n+1)$.

Lemma 2.2 ([24]) Let $f$ be a transcendental meromorphic function of finite order $\rho$. Let $\varepsilon>0$ be a constant, and let $k$ and $j$ be integers satisfying $k>j \geq 0$. Then there exists a set $E_{1} \subset\left[-\frac{\pi}{2}, \frac{3 \pi}{2}\right)$, which has linear measure zero, such that if $\theta \in\left[-\frac{\pi}{2}, \frac{3 \pi}{2}\right) \backslash E_{1}$, then there is a constant $R=R(\theta)>0$ such that for all $z$ satisfying $\arg z=\theta$ and $|z| \geq R$, we have

$$
\left|\frac{f^{(k)}(z)}{f^{(j)}(z)}\right| \leq|z|^{(k-j)(\rho-1+\varepsilon)}
$$

Lemma 2.3 ([17]) Let $g(z)$ be a meromorphic function with $\rho(g)=\beta<\infty$. Then, for any given $\varepsilon>0$, there exists a set $E_{2} \subset\left[-\frac{\pi}{2}, \frac{3 \pi}{2}\right)$ that has linear measure zero such that if $\psi \in$ $\left[-\frac{\pi}{2}, \frac{3 \pi}{2}\right) \backslash E_{2}$, then there is a constant $R=R(\psi)>1$ such that, for all $z$ satisfying $\arg z=\psi$ and $|z|=r>R$, we have

$$
\exp \left\{-r^{\beta+\varepsilon}\right\} \leq|g(z)| \leq \exp \left\{r^{\beta+\varepsilon}\right\}
$$

Lemma 2.4 ([17]) Consider $g(z)=A(z) e^{a z}$, where $A(z)(\not \equiv 0)$ is a meromorphic function with $\rho(A)=\alpha<1, a$ is a complex constant, $a=|a| e^{i \varphi}(\varphi \in[0,2 \pi))$. Set $E_{3}=\{\theta \in[0,2 \pi)$ : $\cos (\varphi+\theta)=0\}$, then $E_{3}$ is a finite set. Then, for any given $\varepsilon(0<\varepsilon<1-\alpha)$, there is a set $E_{4} \in[0,2 \pi)$ that has linear measure zero, if $z=r e^{i \theta}, \theta \in[0,2 \pi) \backslash\left(E_{3} \cup E_{4}\right)$, then we have when $r$ is sufficiently large:

(i) If $\cos (\varphi+\theta)>0$, then

$$
\exp \{(1-\varepsilon) r \delta(a z, \theta)\} \leq|g(z)| \leq \exp \{(1+\varepsilon) r \delta(a z, \theta)\}
$$

(ii) If $\cos (\varphi+\theta)<0$, then

$$
\exp \{(1+\varepsilon) r \delta(a z, \theta)\} \leq|g(z)| \leq \exp \{(1-\varepsilon) r \delta(a z, \theta)\}
$$

where $\delta(a z, \theta)=|a| \cos (\varphi+\theta)$.

Lemma 2.5 ([9]) Suppose that $n \geq 1$ is a positive integer. Let $P_{j}(z)=a_{j n} z^{n}+\cdots(j=1,2)$ be nonconstant polynomials, where $a_{j q}(q=1, \ldots, n)$ are complex numbers and $a_{1 n} a_{2 n} \neq 0$. Set $z=r e^{i \theta}, a_{j n}=\left|a_{j n}\right| e^{i \theta_{j}}, \theta_{j} \in\left[-\frac{\pi}{2}, \frac{3 \pi}{2}\right), \delta\left(P_{j}, \theta\right)=\left|a_{j n}\right| \cos \left(\theta_{j}+n \theta\right)$, then there is a set $E_{5} \subset\left[-\frac{\pi}{2 n}, \frac{3 \pi}{2 n}\right)$ that has linear measure zero. If $\theta_{1} \neq \theta_{2}$, then there exists a ray $\arg z=\theta$, 
$\theta \in\left[-\frac{\pi}{2 n}, \frac{\pi}{2 n}\right) \backslash\left(E_{5} \cup E_{6}\right)$ such that

$$
\delta\left(P_{1}, \theta\right)>0, \quad \delta\left(P_{2}, \theta\right)<0
$$

or

$$
\delta\left(P_{1}, \theta\right)<0, \quad \delta\left(P_{2}, \theta\right)>0,
$$

where $E_{6}=\left\{\theta \in\left[-\frac{\pi}{2 n}, \frac{3 \pi}{2 n}\right): \delta\left(P_{j}, \theta\right)=0\right\}$ is a finite set, which has linear measure zero.

In Lemma 2.5, if $\theta \in\left[-\frac{\pi}{2 n}, \frac{\pi}{2 n}\right) \backslash\left(E_{5} \cup E_{6}\right)$ is replaced by $\theta \in\left[\frac{\pi}{2 n}, \frac{3 \pi}{2 n}\right) \backslash\left(E_{5} \cup E_{6}\right)$, then we can obtain the same result.

Lemma 2.6 ([25]) Let $A_{0}, A_{1}, \ldots, A_{k-1}, F \neq \equiv 0$ be finite order meromorphic functions. Iff $(z)$ is an infinite order meromorphic solution of the equation

$$
f^{(k)}+A_{k-1} f^{(k-1)}+\cdots+A_{1} f^{\prime}+A_{0} f=F,
$$

then $f$ satisfies $\lambda(f)=\bar{\lambda}(f)=\rho(f)=\infty$.

Lemma 2.7 ([26]) Let $k \geq 2$ and $A_{0}, A_{1}, \ldots, A_{k-1}$ be meromorphic functions. Let $\rho=$ $\max \left\{\rho\left(A_{j}\right), j=0,1, \ldots, k-1\right\}$ and all poles of $f$ are of uniformly bounded multiplicity. Then every transcendental meromorphic solution of the differential equation

$$
f^{(k)}+A_{k-1} f^{(k-1)}+\cdots+A_{0} f=0
$$

satisfies $\rho_{2}(f) \leq \rho$.

Remark 2 The condition that the multiplicity of poles of the solution $f$ is uniformly bounded can be changed by $\delta(\infty, f)>0$ for the solution $f$ (see [27]).

Lemma 2.8 (see [24]) Let $f$ be a transcendental meromorphic function. Let $\alpha>1$ be a constant, and let $k$ and $j$ be integers satisfying $k>j \geq 0$. Then there exists a set $E_{7} \subset(1, \infty)$, which has finite logarithmic measure, and a constant $C>0$ such that for all $z$ satisfying $|z| \notin E_{7} \cup[0,1]$, we have (with $r=|z|$ )

$$
\left|\frac{f^{(k)}(z)}{f^{(j)}(z)}\right| \leq C\left[\frac{T(\alpha r, f)}{r}(\log r)^{\alpha} \log T(\alpha r, f)\right]^{k-j}
$$

Lemma 2.9 (see $[2,28]$ ) Let $F(r)$ and $G(r)$ be nondecreasing real-valued functions on $(0, \infty)$ such that $F(r) \leq G(r)$ for all $r$ outside of a set $E \subset(0, \infty)$ of finite linear measure or outside of a set $H \cup[0,1]$, where $H \cup[1, \infty)$ is of finite logarithmic measure. Then, for every constant $\alpha>1$, there exists an $r_{0}>0$ such that $F(r) \leq G(\alpha r)$ for all $r>r_{0}$.

Lemma 2.10 Let $a_{0}$ be a constant satisfying $a_{0}<0$. If $\arg a_{1} \neq \pi$ or $a_{1}<a_{0}$, then we have $a_{1} \neq c a_{0}(0<c \leq 1)$.

The proof is trivial, we omit it here. 


\section{Proof of Theorem 1.1}

First of all we prove that equation (1.2) cannot have a meromorphic solution $f \not \equiv 0$ with $\rho(f)<1$. Assume a meromorphic solution $f \not \equiv 0$ with $\rho(f)=\rho_{1}<1$ satisfies equation (1.2). Then $\rho\left(f^{(j)}\right)=\rho_{1}<1(j=1,2)$. Rewrite $(1.2)$ as

$$
A_{0} f^{\prime} e^{a_{0} z}+A_{1} f e^{a_{1} z}+A_{2} f e^{a_{2} z}=-f^{\prime \prime} .
$$

We consider two cases:

(1) $a_{2} \neq a_{0}$, note that $a_{1} \neq a_{0}, a_{1} \neq a_{2}, \rho\left(A_{1} f\right)<1, \rho\left(A_{2} f\right)<1, \rho\left(A_{0} f^{\prime}\right)<1, \rho\left(-f^{\prime \prime}\right)<1$ and by Lemma 2.1, we have $f \equiv 0$, which is a contradiction.

(2) $a_{2}=a_{0}$, then (3.1) can be rewritten into

$$
A_{1} f e^{a_{1} z}+\left(A_{0} f^{\prime}+A_{2} f\right) e^{a_{2} z}=-f^{\prime \prime}
$$

Note that $a_{1} \neq a_{2}, \rho\left(A_{1} f\right)<1, \rho\left(A_{0} f^{\prime}+A_{2} f\right)<1, \rho\left(-f^{\prime \prime}\right)<1$ and by Lemma 2.1 again, we have $f \equiv 0$, which is a contradiction.

Therefore, $\rho(f) \geq 1$.

Now assume $f$ is a meromorphic solution of equation (1.2) with $1 \leq \rho(f)=\rho<\infty$. From equation (1.2), we know that the poles of $f(z)$ can occur only at the poles of $A_{j}(j=0,1,2)$. Note that the multiplicities of pole points of $f$ are uniformly bounded, and thus we have

$$
N(r, f) \leq M_{1} \bar{N}(r, f) \leq M_{1} \sum_{j=0}^{2} \bar{N}\left(r, A_{j}\right) \leq M \max \left\{N\left(r, A_{j}\right): j=0,1,2\right\},
$$

where $M_{1}$ and $M$ are some suitable positive constants. Then we have $\lambda(1 / f) \leq \alpha=$ $\max \left\{\rho\left(A_{j}\right): j=0,1,2\right\}<1$

Let $f=g / d, d$ be the canonical product formed with the nonzero poles of $f(z)$, with $\beta=\rho(d)=\lambda(d)=\lambda(1 / f) \leq \alpha<1$, let $g$ be an entire function and $1 \leq \rho(g)=\rho(f)=\rho<\infty$. Substituting $f=g / d$ into (1.2), we can get

$$
\frac{g^{\prime \prime}}{g}+\left[A_{0} e^{a_{0} z}-2 \frac{d^{\prime}}{d}\right] \frac{g^{\prime}}{g}+2\left(\frac{d^{\prime}}{d}\right)^{2}-\frac{d^{\prime \prime}}{d}-A_{0} \frac{d^{\prime}}{d} e^{a_{0} z}+A_{1} e^{a_{1} z}+A_{2} e^{a_{2} z}=0
$$

For any given $\varepsilon(0<\varepsilon<1-\alpha)$, there is a set $E_{1} \subset\left[-\frac{\pi}{2}, \frac{3 \pi}{2}\right)$ that has linear measure zero such that if $\theta \in\left[-\frac{\pi}{2}, \frac{3 \pi}{2}\right) \backslash E_{1}$, then there is a constant $R=R(\theta)>1$ such that for all $z$ satisfying $\arg z=\theta$, and $|z|=r>R$, we have by Lemma 2.3

$$
\left|A_{0}(z)\right| \leq \exp \left\{r^{\alpha+\varepsilon}\right\}
$$

By Lemma 2.2, for any given $\varepsilon\left(0<\varepsilon<\min \left\{1-\alpha, \frac{\left|a_{2}\right|-\left|a_{1}\right|}{\left.\left|a_{2}\right|+\mid a_{1}\right\}}\right)\right.$, there exists a set $E_{2} \subset\left[-\frac{\pi}{2}, \frac{3 \pi}{2}\right)$ that has linear measure zero such that if $\theta \in\left[-\frac{\pi}{2}, \frac{3 \pi}{2}\right) \backslash E_{2}$, then there is a constant $R_{0}=$ $R_{0}(\theta)>1$ such that for all $z$ satisfying $\arg z=\theta$ and $|z| \geq R_{0}$, we have

$$
\left|\frac{g^{(j)}(z)}{g(z)}\right| \leq|z|^{j(\rho-1+\varepsilon)}, \quad j=1,2,
$$


and

$$
\left|\frac{d^{(j)}(z)}{d(z)}\right| \leq|z|^{j(\beta-1+\varepsilon)}, \quad j=1,2 .
$$

Setting $z=r e^{i \theta}, a_{1}=\left|a_{1}\right| e^{i \theta_{1}}, a_{2}=\left|a_{2}\right| e^{i \theta_{2}}, \theta_{1}, \theta_{2} \in\left[-\frac{\pi}{2}, \frac{3 \pi}{2}\right)$.

Case $1 . \arg a_{1} \neq \pi$, which is $\theta_{1} \neq \pi$.

Subcase 1.1. Assume that $\theta_{1} \neq \theta_{2}$. By Lemma 2.5, for the above $\varepsilon$, there is a ray $\arg z=\theta$ such that $\theta \in\left(-\frac{\pi}{2}, \frac{\pi}{2}\right) \backslash\left(E_{1} \cup E_{2} \cup E_{5} \cup E_{6}\right)$ (where $E_{5}$ and $E_{6}$ are defined as in Lemma 2.5, $E_{1} \cup$ $E_{2} \cup E_{5} \cup E_{6}$ is of linear measure zero) satisfying $\delta\left(a_{1} z, \theta\right)>0, \delta\left(a_{2} z, \theta\right)<0$ or $\delta\left(a_{1} z, \theta\right)<0$, $\delta\left(a_{2} z, \theta\right)>0$ for a sufficiently large $r$.

When $\delta\left(a_{1} z, \theta\right)>0, \delta\left(a_{2} z, \theta\right)<0$ for a sufficiently large $r$, we have, by Lemma 2.4,

$$
\begin{aligned}
& \left|A_{1} e^{a_{1} z}\right| \geq \exp \left\{(1-\varepsilon) \delta\left(a_{1} z, \theta\right) r\right\}, \\
& \left|A_{2} e^{a_{2} z}\right| \leq \exp \left\{(1-\varepsilon) \delta\left(a_{2} z, \theta\right) r\right\}<1 .
\end{aligned}
$$

By (3.6) and (3.7), we have

$$
\begin{aligned}
\left|A_{1} e^{a_{1} z}+A_{2} e^{a_{2} z}\right| & \geq\left|A_{1} e^{a_{1} z}\right|-\left|A_{2} e^{a_{2} z}\right| \geq \exp \left\{(1-\varepsilon) \delta\left(a_{1} z, \theta\right) r\right\}-1 \\
& =(1-o(1)) \exp \left\{(1-\varepsilon) \delta\left(a_{1} z, \theta\right) r\right\} .
\end{aligned}
$$

By (3.2), we get

$$
\begin{aligned}
\left|A_{1} e^{a_{1} z}+A_{2} e^{a_{2} z}\right| \leq & \left|\frac{g^{\prime \prime}}{g}\right|+\left(\left|A_{0} e^{a_{0} z}\right|+2\left|\frac{d^{\prime}}{d}\right|\right)\left|\frac{g^{\prime}}{g}\right| \\
& +2\left|\frac{d^{\prime}}{d}\right|^{2}+\left|\frac{d^{\prime \prime}}{d}\right|+\left|A_{0}\right|\left|\frac{d^{\prime}}{d}\right|\left|e^{a_{0} z}\right|
\end{aligned}
$$

Since $\theta \in\left(-\frac{\pi}{2}, \frac{\pi}{2}\right) \backslash\left(E_{1} \cup E_{2} \cup E_{5} \cup E_{6}\right)$, we know that $\cos \theta>0$, then $\left|e^{a_{0} z}\right|=e^{-\left|a_{0}\right| r \cos \theta}<1$. Therefore, by (3.3) we obtain

$$
\left|A_{0}(z) e^{a_{0} z}\right| \leq \exp \left\{r^{\alpha+\varepsilon}\right\}
$$

Substituting (3.4)-(3.5), (3.8) and (3.10) into (3.9), we obtain

$$
(1-o(1)) \exp \left\{(1-\varepsilon) \delta\left(a_{1} z, \theta\right) r\right\} \leq M_{1} r^{k(\rho-1+\varepsilon)} \exp \left\{r^{\alpha+\varepsilon}\right\}
$$

where $M_{1}>0$ and $k>0$ are some constants. By $\delta\left(a_{1} z, \theta\right)>0$ and $\alpha+\varepsilon<1$, we know that (3.11) is a contradiction.

When $\delta\left(a_{1} z, \theta\right)<0, \delta\left(a_{2} z, \theta\right)>0$, using a proof similar to the above, we can get a contradiction.

Subcase 1.2. Assume that $\theta_{1}=\theta_{2}$. By Lemma 2.5, for the above $\varepsilon$, there is a ray $\arg z=\theta$ such that $\theta \in\left(-\frac{\pi}{2}, \frac{\pi}{2}\right) \backslash\left(E_{1} \cup E_{2} \cup E_{5} \cup E_{6}\right)$ satisfying $\delta\left(a_{1} z, \theta\right)>0$. Since $\left|a_{1}\right| \leq\left|a_{2}\right|, a_{1} \neq a_{2}$ and $\theta_{1}=\theta_{2}$, then $\left|a_{1}\right|<\left|a_{2}\right|$, thus $\delta\left(a_{2} z, \theta\right)>\delta\left(a_{1} z, \theta\right)>0$. For a sufficiently large $r$, we get, 
by Lemma 2.4 ,

$$
\begin{aligned}
& \left|A_{1} e^{a_{1} z}\right| \leq \exp \left\{(1+\varepsilon) \delta\left(a_{1} z, \theta\right) r\right\} \\
& \left|A_{2} e^{a_{2} z}\right| \geq \exp \left\{(1-\varepsilon) \delta\left(a_{2} z, \theta\right) r\right\} .
\end{aligned}
$$

By (3.12) and (3.13), we get

$$
\begin{aligned}
\left|A_{1} e^{a_{1} z}+A_{2} e^{a_{2} z}\right| & \geq\left|A_{2} e^{a_{2} z}\right|-\left|A_{1} e^{a_{1} z}\right| \\
& \geq \exp \left\{(1-\varepsilon) \delta\left(a_{2} z, \theta\right) r\right\}-\exp \left\{(1+\varepsilon) \delta\left(a_{1} z, \theta\right) r\right\} \\
& =M_{2} \exp \left\{(1+\varepsilon) \delta\left(a_{1} z, \theta\right) r\right\},
\end{aligned}
$$

where $M_{2}=\exp \left\{\left[(1-\varepsilon) \delta\left(a_{2} z, \theta\right)-(1+\varepsilon) \delta\left(a_{1} z, \theta\right)\right] r\right\}-1$.

Since $0<\varepsilon<\min \left\{1-\alpha, \frac{\left|a_{2}\right|-\left|a_{1}\right|}{\left|a_{2}\right|+\left|a_{1}\right|}\right\}$, we see that $(1-\varepsilon) \delta\left(a_{2} z, \theta\right)-(1+\varepsilon) \delta\left(a_{1} z, \theta\right)>0$, then $\exp \left\{\left[(1-\varepsilon) \delta\left(a_{2} z, \theta\right)-(1+\varepsilon) \delta\left(a_{1} z, \theta\right)\right] r\right\}>1, M_{2}>0$.

Since $\theta \in\left(-\frac{\pi}{2}, \frac{\pi}{2}\right) \backslash\left(E_{1} \cup E_{2} \cup E_{5} \cup E_{6}\right)$, we know that $\cos \theta>0$, then $\left|e^{a_{0} z}\right|=e^{-\left|a_{0}\right| r \cos \theta}<1$. Therefore, by (3.3) we obtain

$$
\left|A_{0}(z) e^{a_{0} z}\right| \leq \exp \left\{r^{\alpha+\varepsilon}\right\}
$$

Substituting (3.4)-(3.5) and (3.14)-(3.15) into (3.9), we obtain

$$
M_{2} \exp \left\{(1+\varepsilon) \delta\left(a_{1} z, \theta\right) r\right\} \leq M_{1} r^{k(\rho-1+\varepsilon)} \exp \left\{r^{\alpha+\varepsilon}\right\}
$$

By $\delta\left(a_{1} z, \theta\right)>0, M_{2}>0$ and $\alpha+\varepsilon<1$, we know that (3.16) is a contradiction.

Case 2. $a_{1}<a_{0}$, which is $\theta_{1}=\pi$.

Subcase 2.1. Assume that $\theta_{1} \neq \theta_{2}$, then $\theta_{2} \neq \pi$. By Lemma 2.5, for the above $\varepsilon$, there is a ray $\arg z=\theta$ such that $\theta \in\left(-\frac{\pi}{2}, \frac{\pi}{2}\right) \backslash\left(E_{1} \cup E_{2} \cup E_{5} \cup E_{6}\right)$ satisfying $\delta\left(a_{2} z, \theta\right)>0$. Since $\cos \theta>0$, we have $\delta\left(a_{1} z, \theta\right)=\left|a_{1}\right| \cos \left(\theta_{1}+\theta\right)=-\left|a_{1}\right| \cos \theta<0$. For a sufficiently large $r$, we have

$$
\begin{aligned}
& \left|A_{1} e^{a_{1} z}\right| \leq \exp \left\{(1-\varepsilon) \delta\left(a_{1} z, \theta\right) r\right\}<1, \\
& \left|A_{2} e^{a_{2} z}\right| \geq \exp \left\{(1-\varepsilon) \delta\left(a_{2} z, \theta\right) r\right\} .
\end{aligned}
$$

By (3.17) and (3.18), we get

$$
\begin{aligned}
\left|A_{1} e^{a_{1} z}+A_{2} e^{a_{2} z}\right| & \geq\left|A_{2} e^{a_{2} z}\right|-\left|A_{1} e^{a_{1} z}\right| \\
& \geq \exp \left\{(1-\varepsilon) \delta\left(a_{2} z, \theta\right) r\right\}-1
\end{aligned}
$$

Using the same reasoning as in Subcase 1.1, we can get a contradiction.

Subcase 2.2. Assume that $\theta_{1}=\theta_{2}$, then $\theta_{1}=\theta_{2}=\pi$. By Lemma 2.5, for the above $\varepsilon$, there is a ray $\arg z=\theta$ such that $\theta \in\left(\frac{\pi}{2}, \frac{3 \pi}{2}\right) \backslash\left(E_{1} \cup E_{2} \cup E_{5} \cup E_{6}\right)$, then $\cos \theta<0, \delta\left(a_{1} z, \theta\right)=$ $\left|a_{1}\right| \cos \left(\theta_{1}+\theta\right)=-\left|a_{1}\right| \cos \theta>0, \delta\left(a_{2} z, \theta\right)=\left|a_{2}\right| \cos \left(\theta_{1}+\theta\right)=-\left|a_{2}\right| \cos \theta>0$. Since $\left|a_{1}\right| \leq$ $\left|a_{2}\right|, a_{1} \neq a_{2}$ and $\theta_{1}=\theta_{2}$, then $\left|a_{1}\right|<\left|a_{2}\right|$, thus $\delta\left(a_{2} z, \theta\right)>\delta\left(a_{1} z, \theta\right)>0$. For a sufficiently large $r$, we get (3.12), (3.13) and (3.14) hold.

Using the same reasoning as in Subcase 1.2, we can get a contradiction. 
Concluding the above proof, we obtain $\rho(f)=\rho(g)=+\infty$.

In the following, we prove that if all poles of $f$ are of uniformly bounded multiplicity, then $\rho_{2}(f)=1$.

By Lemma 2.7 and $\max \left\{\rho\left(A_{0} e^{a_{0} z}\right), \rho\left(A_{1} e^{a_{1} z}+A_{2} e^{a_{2} z}\right)\right\}=1$, then $\rho_{2}(f) \leq 1$.

By Lemma 2.8, we know that there exists a set $E_{7} \subset(1,+\infty)$ with finite logarithmic measure and a constant $C>0$ such that for all $z$ satisfying $|z|=r \notin[0,1] \cup E_{7}$, we get

$$
\left|\frac{f^{(j)}(z)}{f(z)}\right| \leq C[T(2 r, f) \log T(2 r, f)]^{j} \leq C[T(2 r, f)]^{j+1}, \quad j=1,2 .
$$

For Subcases 1.1 and 2.1, we have proved that there exists a ray $\arg z=\theta$ satisfying $\theta \in$ $\left(-\frac{\pi}{2}, \frac{\pi}{2}\right) \backslash\left(E_{1} \cup E_{2} \cup E_{5} \cup E_{6}\right)$, for a sufficiently large $r$, we get that (3.8) or (3.19) hold, that is,

$$
\left|A_{1} e^{a_{1} z}+A_{2} e^{a_{2} z}\right| \geq \exp \left\{h_{1} r\right\}
$$

where $h_{1}>0$ is a constant.

By (1.2), we have

$$
\frac{f^{\prime \prime}}{f}+A_{0} e^{a_{0} z} \frac{f^{\prime}}{f}=-\left(A_{1} e^{a_{1} z}+A_{2} e^{a_{2} z}\right) .
$$

Since $\theta \in\left(-\frac{\pi}{2}, \frac{\pi}{2}\right) \backslash\left(E_{1} \cup E_{2} \cup E_{5} \cup E_{6}\right)$, then $\cos \theta>0, e^{-\left|a_{0}\right| r \cos \theta}<1$. By (3.20), (3.21) and (3.22), we obtain

$$
\begin{aligned}
\exp \left\{h_{1} r\right\} & \leq C[T(2 r, f)]^{3}+\exp \left\{r^{\alpha+\varepsilon}\right\} e^{-\left|a_{0}\right| r \cos \theta} C[T(2 r, f)]^{2} \\
& \leq 2 C \exp \left\{r^{\alpha+\varepsilon}\right\}[T(2 r, f)]^{3} .
\end{aligned}
$$

By $h_{1}>0, \alpha+\varepsilon<1$, (3.23) and Lemma 2.9, we know that for the constant $\alpha=2$, there exists $r_{0}$. When $r>r_{0}$, we have $\rho_{2}(f) \geq 1$, then $\rho_{2}(f)=1$.

For Subcases 1.2 and 2.2, we have proved that there exists a ray $\arg z=\theta$ such that $\theta \in$ $\left(\frac{\pi}{2}, \frac{3 \pi}{2}\right) \backslash\left(E_{1} \cup E_{2} \cup E_{5} \cup E_{6}\right)$. For a sufficiently large $r$, we get that (3.14) holds, and we also get $\cos \theta<0, \delta\left(a_{1} z, \theta\right)>-\left|a_{0}\right| \cos \theta>0$.

By (3.14), (3.20) and (3.22), we obtain

$$
\begin{aligned}
M_{2} \exp \left\{(1+\varepsilon) \delta\left(a_{1} z, \theta\right) r\right\} & \leq C[T(2 r, f)]^{3}+\exp \left\{r^{\alpha+\varepsilon}\right\} e^{-\left|a_{0}\right| r \cos \theta} C[T(2 r, f)]^{2} \\
& \leq 2 C e^{-\left|a_{0}\right| r \cos \theta} \exp \left\{r^{\alpha+\varepsilon}\right\}[T(2 r, f)]^{3} .
\end{aligned}
$$

By $\delta\left(a_{1} z, \theta\right)>-\left|a_{0}\right| \cos \theta>0, M_{2}>0, \alpha+\varepsilon<1$ and (3.24) and Lemma 2.9, we know that for the constant $\alpha=2$, there exists $r_{0}$, when $r>r_{0}$, we have $\rho_{2}(f) \geq 1$, then $\rho_{2}(f)=1$.

The proof of Theorem 1.1 is complete.

\section{Proof of Theorem 1.2}

Assume that $f(\not \equiv 0)$ is a meromorphic solution of (1.2); then $\rho(f)=\infty$ by Theorem 1.1. Set $g_{0}(z)=f(z)-\varphi(z), g_{0}(z)$ is a meromorphic function and $\rho\left(g_{0}\right)=\rho(f)=\infty$. Substituting $f=g_{0}+\varphi$ into (1.2), we have

$$
g_{0}^{\prime \prime}+h_{0,1} g_{0}^{\prime}+h_{0,0} g_{0}=h_{0}
$$


where $h_{0,1}=A_{0} e^{a_{0} z}, h_{0,0}=A_{1} e^{a_{1} z}+A_{2} e^{a_{2} z}$ and $h_{0}=-\left(\varphi^{\prime \prime}+h_{0,1} \varphi^{\prime}+h_{0,0} \varphi\right)$. Obviously, $h_{0} \not \equiv 0$. In fact, if $h_{0} \equiv 0$, then

$$
\varphi^{\prime \prime}+h_{0,1} \varphi^{\prime}+h_{0,0} \varphi=0 .
$$

By Theorem 1.1, we have $\rho(\varphi)=\infty$, it is a contradiction. Hence $h_{0} \not \equiv 0$. Note that the functions $h_{0,1}, h_{0,0}$ and $h_{0}$ are of finite order, by Lemma 2.6 and (4.1), we have $\bar{\lambda}\left(g_{0}\right)=$ $\bar{\lambda}(f-\varphi)=\infty$.

Now we prove that $\bar{\lambda}\left(f^{\prime}-\varphi\right)=\infty$. Let $g_{1}(z)=f^{\prime}(z)-\varphi(z)$, then $\rho\left(g_{1}\right)=\rho\left(f^{\prime}\right)=\rho(f)=\infty$ and $\bar{\lambda}\left(g_{1}\right)=\bar{\lambda}\left(f^{\prime}-\varphi\right)$.

Differentiating both sides of (1.2), we have

$$
f^{\prime \prime \prime}+h_{0,1} f^{\prime \prime}+\left[h_{0,1}^{\prime}+h_{0,0}\right] f^{\prime}+h_{0,0}^{\prime} f=0 .
$$

By (1.2), we have

$$
f=-\frac{1}{h_{0,0}}\left(f^{\prime \prime}+h_{0,1} f^{\prime}\right) .
$$

Substituting (4.3) into (4.2), we have

$$
f^{\prime \prime \prime}+\left(h_{0,1}-\frac{h_{0,0}^{\prime}}{h_{0,0}}\right) f^{\prime \prime}+\left[h_{0,1}^{\prime}+h_{0,0}-\frac{h_{0,0}^{\prime}}{h_{0,0}} h_{0,1}\right] f^{\prime}=0 .
$$

Combining $f^{\prime}=g_{1}+\varphi, f^{\prime \prime}=g_{1}^{\prime}+\varphi^{\prime}, f^{\prime \prime \prime}=g_{1}^{\prime \prime}+\varphi^{\prime \prime}$ into (4.4), we have

$$
g_{1}^{\prime \prime}+h_{1,1} g_{1}^{\prime}+h_{1,0} g_{1}=h_{1},
$$

where

$$
\begin{aligned}
& h_{1,1}=h_{0,1}-\frac{h_{0,0}^{\prime}}{h_{0,0}}, \\
& h_{1,0}=h_{0,1}^{\prime}+h_{0,0}-\frac{h_{0,0}^{\prime}}{h_{0,0}} h_{0,1}, \\
& -h_{1}=\varphi^{\prime \prime}+\left(h_{0,1}-\frac{h_{0,0}^{\prime}}{h_{0,0}}\right) \varphi^{\prime}+\left[h_{0,1}^{\prime}+h_{0,0}-\frac{h_{0,0}^{\prime}}{h_{0,0}} h_{0,1}\right] \varphi .
\end{aligned}
$$

Now we prove $h_{1} \not \equiv 0$. In fact, if $h_{1} \equiv 0$, then

$$
\begin{aligned}
& {\left[\left(\frac{\varphi^{\prime}}{\varphi} A_{0}+A_{0}^{\prime}+a_{0} A_{0}-a_{1} A_{0}\right) A_{1}-A_{0} A_{1}^{\prime}\right] e^{\left(a_{0}+a_{1}\right) z}} \\
& \quad+\left[\left(\frac{\varphi^{\prime}}{\varphi} A_{0}+A_{0}^{\prime}+a_{0} A_{0}-a_{2} A_{0}\right) A_{2}-A_{0} A_{2}^{\prime}\right] e^{\left(a_{0}+a_{2}\right) z} \\
& \quad+\left[\left(\frac{\varphi^{\prime \prime}}{\varphi}-a_{1} \frac{\varphi^{\prime}}{\varphi}\right) A_{1}-\frac{\varphi^{\prime}}{\varphi} A_{1}^{\prime}\right] e^{a_{1} z}+\left[\left(\frac{\varphi^{\prime \prime}}{\varphi}-a_{2} \frac{\varphi^{\prime}}{\varphi}\right) A_{2}-\frac{\varphi^{\prime}}{\varphi} A_{2}^{\prime}\right] e^{a_{2} z} \\
& \quad+2 A_{1} A_{2} e^{\left(a_{1}+a_{2}\right) z}+A_{1}^{2} e^{2 a_{1} z}+A_{2}^{2} e^{2 a_{2} z} \equiv 0 .
\end{aligned}
$$


We can rewrite (4.6) in the form

$$
f_{1} e^{\left(a_{0}+a_{1}\right) z}+f_{2} e^{\left(a_{0}+a_{2}\right) z}+f_{3} e^{a_{1} z}+f_{4} e^{a_{2} z}+f_{5} e^{\left(a_{1}+a_{2}\right) z}+f_{6} e^{2 a_{1} z}+f_{7} e^{2 a_{2} z} \equiv 0 .
$$

Since $\rho=\rho(\varphi)<1, \rho\left(A_{k}\right)<1(k=0,1,2)$, then $\rho\left(f_{j}\right)<1(j=1, \ldots, 7)$. Note that $a_{1} \neq a_{2}$ and Lemma 2.10 , then $2 a_{1} \neq a_{1}, a_{1}+a_{0}, a_{1}+a_{2}, 2 a_{2}$. In order to apply Lemma 2.1 , we need to consider three cases:

(i) If $2 a_{1} \neq a_{0}+a_{2}, a_{2}$. By Lemma 2.1, we get $f_{6} \equiv 0$, that is, $A_{1} \equiv 0$, a contradiction.

(ii) If $2 a_{1}=a_{0}+a_{2}$, then by Lemma 2.10, $2 a_{2} \neq a_{1}, 2 a_{1}, a_{2}+a_{0}, a_{1}+a_{2}, a_{0}+a_{1}, a_{2}$. By Lemma 2.1 , we get $f_{7} \equiv 0$, that is, $A_{2} \equiv 0$, a contradiction.

(iii) If $2 a_{1}=a_{2}$, then by Lemma 2.10, $2 a_{2} \neq a_{1}, 2 a_{1}, a_{2}+a_{0}, a_{1}+a_{2}, a_{0}+a_{1}, a_{2}$. By Lemma 2.1 , we get $f_{7} \equiv 0$, that is, $A_{2} \equiv 0$, a contradiction.

Hence $h_{1} \neq 0$.

For equation (4.5), since $h_{1} \not \equiv 0$ and $\rho\left(g_{1}\right)=\infty$, by Lemma 2.6, we have

$$
\bar{\lambda}\left(g_{1}\right)=\bar{\lambda}\left(f^{\prime}-\varphi\right)=\rho\left(g_{1}\right)=\rho(f)=\infty .
$$

Using a similar way to above, we can easily prove that $h_{1,0} \not \equiv 0$. We omit it here. In the following, we prove $\bar{\lambda}\left(f^{\prime \prime}-\varphi\right)=\infty$.

Let $g_{2}(z)=f^{\prime \prime}-\varphi$, then $\rho\left(g_{2}\right)=\rho\left(f^{\prime \prime}\right)=\rho(f)=\infty$ and $\bar{\lambda}\left(g_{2}\right)=\bar{\lambda}\left(f^{\prime \prime}-\varphi\right)$.

Differentiating the two sides of (1.2), we get

$$
f^{(4)}+h_{0,1} f^{\prime \prime \prime}+\left[2 h_{0,1}^{\prime}+h_{0,0}\right] f^{\prime \prime}+\left[h_{0,1}^{\prime \prime}+2 h_{0,0}^{\prime}\right] f^{\prime}+h_{0,0}^{\prime \prime} f=0 .
$$

By (4.4), we have

$$
f^{\prime}=\frac{-1}{h_{1,0}}\left[f^{\prime \prime \prime}+h_{1,1} f^{\prime \prime}\right] .
$$

From (4.3), (4.8) and (4.9), we obtain

$$
f^{(4)}+h_{2,1} f^{\prime \prime \prime}+h_{2,0} f^{\prime \prime}=0
$$

where

$$
\begin{aligned}
& h_{2,1}=h_{0,1}-\frac{\varphi_{1}}{h_{1,0}}, \\
& h_{2,0}=2 h_{0,1}^{\prime}+h_{0,0}-\frac{h_{0,0}^{\prime \prime}}{h_{0,0}}-\frac{\varphi_{1}}{h_{1,0}}\left[h_{0,1}-\frac{h_{0,0}^{\prime}}{h_{0,0}}\right],
\end{aligned}
$$

where

$$
\varphi_{1}=h_{0,1}^{\prime \prime}+2 h_{0,0}^{\prime}-h_{0,1} \frac{h_{0,0}^{\prime \prime}}{h_{0,0}} .
$$

Substituting $f^{\prime \prime}=g_{2}+\varphi, f^{\prime \prime \prime}=g_{2}^{\prime}+\varphi^{\prime}, f^{(4)}=g_{2}^{\prime \prime}+\varphi^{\prime \prime}$ into (4.10), we have

$$
g_{2}^{\prime \prime}+h_{2,1} g_{2}^{\prime}+h_{2,0} g_{2}=h_{2}
$$


where

$$
h_{2}=-\left(\varphi^{\prime \prime}+h_{2,1} \varphi^{\prime}+h_{2,0} \varphi\right) \text {. }
$$

Similarly, if one can prove $h_{2} \not \equiv 0$, then by Lemma 2.6, we can get $\bar{\lambda}\left(g_{2}\right)=\rho\left(g_{2}\right)=\infty$. Hence $\bar{\lambda}\left(f^{\prime \prime}-\varphi\right)=\infty$.

Now we prove $h_{2} \not \equiv 0$. Note that

$$
\begin{aligned}
& h_{2,1}=\frac{H_{1}}{h_{1,0} h_{0,0}}, \\
& h_{2,0}=\frac{H_{2}}{h_{1,0} h_{0,0}},
\end{aligned}
$$

where

$$
\begin{aligned}
H_{1}= & h_{0,0} h_{0,1} h_{0,1}^{\prime}+h_{0,0}^{2} h_{0,1}-h_{0,0}^{\prime} h_{0,1}^{2}-h_{0,0} h_{0,1}^{\prime \prime}-2 h_{0,0} h_{0,0}^{\prime}+h_{0,1} h_{0,0}^{\prime \prime}, \\
H_{2}= & 2 h_{0,1}^{\prime 2} h_{0,0}+3 h_{0,0}^{2} h_{0,1}-2 h_{0,0} h_{0,1} h_{0,1}^{\prime}+h_{0,0}^{3}-3 h_{0,0} h_{0,0}^{\prime} h_{0,1}-h_{0,1}^{\prime} h_{0,0}^{\prime \prime} \\
& -h_{0,0}^{\prime \prime} h_{0,0}-h_{0,1} h_{0,1}^{\prime \prime} h_{0,0}+2 h_{0,0}^{\prime 2}+h_{0,0}^{\prime} h_{0,1}^{\prime \prime}+h_{0,1}^{2} h_{0,0}^{\prime \prime} .
\end{aligned}
$$

Obviously, $H_{1}, H_{2}$ are meromorphic functions and $\rho\left(H_{j}\right)<1(j=1,2)$. From (4.14), (4.15)(4.16), we know

$$
\frac{h_{2}}{\varphi}=-\frac{1}{h_{1,0} h_{0,0}}\left[\frac{\varphi^{\prime \prime}}{\varphi} h_{1,0} h_{0,0}+\frac{\varphi^{\prime}}{\varphi} H_{1}+H_{2}\right] .
$$

Let $H=\frac{\varphi^{\prime \prime}}{\varphi} h_{1,0} h_{0,0}+\frac{\varphi^{\prime}}{\varphi} H_{1}+H_{2}$. We only need to prove $H \not \equiv 0$.

Note that $\rho\left(\frac{\varphi^{\prime \prime}}{\varphi}\right)<1, \rho\left(\frac{\varphi^{\prime}}{\varphi}\right)<1$ and $h_{1,0} h_{0,0}=h_{0,1}^{\prime} h_{0,0}+h_{0,0}^{2}-h_{0,1} h_{0,0}^{\prime}$. From this and (4.15)(4.16), we can write $H$ into the following form:

$$
\begin{aligned}
H= & f_{1} e^{\left(a_{0}+a_{1}\right) z}+f_{2} e^{\left(a_{0}+a_{2}\right) z}+f_{3} e^{2 a_{1} z}+f_{4} e^{2 a_{2} z}+f_{5} e^{\left(a_{1}+a_{2}\right) z} \\
& +f_{6} e^{\left(2 a_{1}+a_{2}\right) z}+f_{7} e^{\left(a_{1}+2 a_{2}\right) z}+f_{8} e^{\left(2 a_{0}+a_{1}\right) z}+f_{9} e^{\left(2 a_{0}+a_{2}\right) z}+f_{10} e^{\left(2 a_{1}+a_{0}\right) z} \\
& +f_{11} e^{\left(2 a_{2}+a_{0}\right) z}+f_{12} e^{\left(a_{0}+a_{1}+a_{2}\right) z}+f_{13} e^{3 a_{1} z}+f_{14} e^{3 a_{2} z} .
\end{aligned}
$$

It is easy to see $f_{13}=A_{1}^{3}, f_{14}=A_{2}^{3}$, which come from the term $h_{0,0}^{3}$ in $H_{2}$.

We can prove that $\rho\left(f_{i}\right)<1(1 \leq i \leq 12)$. Set $\Lambda=\left\{a_{0}+a_{1}+a_{2}, a_{0}+a_{2}, 2 a_{1}, 2 a_{2}, a_{1}+a_{2}, 2 a_{1}+\right.$ $\left.a_{2}, a_{1}+2 a_{2}, 2 a_{0}+a_{1}, 2 a_{0}+a_{2}, 2 a_{1}+a_{0}, 2 a_{2}+a_{0}, a_{1}+a_{2}, 3 a_{1}, 3 a_{2}\right\}$. Note that $a_{1} \neq a_{2}$ and Lemma 2.10, then $3 a_{1} \neq a_{1}+a_{0}, a_{1}+2 a_{0}, 2 a_{1}, 2 a_{1}+a_{0}, 2 a_{1}+a_{2}, 3 a_{2}, a_{1}+2 a_{2}$ and $3 a_{2} \neq$ $2 a_{2}, 3 a_{1}, 2 a_{1}+a_{2}, a_{1}+2 a_{2}$. Using the same way as above, we need to consider seven cases:

(i) If $3 a_{1} \neq a_{2}+a_{0}, a_{2}+2 a_{0}, a_{1}+a_{2}, 2 a_{2}+a_{0}, a_{0}+a_{1}+a_{2}, 2 a_{2}$. By Lemma 2.1, we get $f_{13} \equiv 0$, that is, $A_{1} \equiv 0$, a contradiction.

(ii) If $3 a_{1}=a_{2}+a_{0}$, then we can conclude that $3 a_{2} \neq \Lambda-\left\{3 a_{2}\right\}$. Hence, by Lemma 2.1, we get $f_{14} \equiv 0$, that is, $A_{2} \equiv 0$, a contradiction.

(iii) If $3 a_{1}=a_{2}+2 a_{0}$, then we can conclude that $3 a_{2} \neq \Lambda-\left\{3 a_{2}\right\}$. Hence, by Lemma 2.1, we get $f_{14} \equiv 0$, that is, $A_{2} \equiv 0$, a contradiction.

(iv) If $3 a_{1}=a_{1}+a_{2}$, then we can conclude that $3 a_{2} \neq \Lambda-\left\{3 a_{2}\right\}$. Hence, by Lemma 2.1, we get $f_{14} \equiv 0$, that is, $A_{2} \equiv 0$, a contradiction. 
(v) If $3 a_{1}=2 a_{2}+a_{0}$, then we can conclude that $3 a_{2} \neq \Lambda-\left\{3 a_{2}\right\}$. Hence, by Lemma 2.1, we get $f_{14} \equiv 0$, that is, $A_{2} \equiv 0$, a contradiction.

(vi) If $3 a_{1}=a_{0}+a_{1}+a_{2}$, then we can conclude that $3 a_{2} \neq \Lambda-\left\{3 a_{2}\right\}$. Hence, by Lemma 2.1, we get $f_{14} \equiv 0$, that is, $A_{2} \equiv 0$, a contradiction.

(vii) If $3 a_{1}=2 a_{2}$, then we conclude that $3 a_{2} \neq \Lambda-\left\{3 a_{2}\right\}$. Hence, by Lemma 2.1, we get $f_{14} \equiv 0$, that is, $A_{2} \equiv 0$, a contradiction.

Hence, we prove $H \not \equiv 0$ and $h_{2} \not \equiv 0$.

\section{This proved the theorem.}

\section{Competing interests}

The authors declare that they have no competing interests.

\section{Authors' contributions}

All authors drafted the manuscript, read and approved the final manuscript.

\section{Author details}

${ }^{1}$ Department of Mathematics, Wuyi University, Jiangmen, Guangdong 529020, P.R. China. ${ }^{2}$ College of Science, Civil Aviation University of China, Tianjin, 300300, P.R. China.

\section{Acknowledgements}

The authors would like to thank the referee for his/her constructive suggestions and for pointing out some errors in our original manuscript. These comments greatly improved the readability of the paper. This work was supported by the National Natural Science Foundation of China (No. 11126327, 11171184), NSF of Guangdong Province (No. S2011010000735), STP of Jiangmen, China (No. [2011]133) and the Outstanding Young Innovative Talents Fund of Department of Education of Guangdong (No. 2012LYM0126).

Received: 15 August 2012 Accepted: 9 June 2013 Published: 2 July 2013

\section{References}

1. Hayman, W: Meromorphic Functions. Clarendon, Oxford (1964)

2. Laine, I: Nevanlinna Theory and Complex Differential Equations. de Gruyter, Berlin (1993)

3. Yang, CC, Yi, HX: The Uniqueness Theory of Meromorphic Functions. Kluwer Academic, New York (2003)

4. Chen, ZX: The fixed points and hyper-order of solutions of second order linear differential equations. Acta Math. Sci. 20, 425-432 (2000) (in Chinese)

5. Zhang, XM, Liu, MS: The frequency of the zeros of the difference of a solution to a linear differential equation and a small function. Southeast Asian Bull. Math. 33, 999-1011 (2009)

6. Frei, M: Über die subnormalen Lösungen der Differentialgleichungen $w^{\prime \prime}+e^{-z} w^{\prime}+($ konst.) $f=0$. Comment. Math. Helv. 36, 1-8 (1961)

7. Gundersen, $\mathrm{G}$ : On the question of whether $f^{\prime \prime}+e^{-z} f^{\prime}+B(z) f=0$ can admit a solution $f \not \equiv 0$ of finite order. Proc. R. Soc. Edinb., Sect. A 102, 9-17 (1986)

8. Chen, ZX: The growth of solutions of differential equation $f^{\prime \prime}+e^{-z} f^{\prime}+Q(z) f=0$. Sci. China Ser. A 31(9), 775-784 (2001)

9. Peng, F, Chen, ZX: On the growth of solutions of some second-order linear differential equations. J. Inequal. Appl. 2011, Article ID 635604 (2011)

10. Bank, SB: A note on algebraic differential equations whose coefficients are entire functions of finite order. Ann. Sc. Norm. Super. Pisa, Cl. Sci. 83, 291-297 (1972)

11. Bank, SB: On the existence of meromorphic solutions of differential equations having arbitrarily rapid growth. J. Reine Angew. Math. 288, 176-182 (1976)

12. Bank, SB, Laine, I: On the growth of meromorphic solutions of linear and algebraic differential equations. Math. Scand. 40, 119-126 (1977)

13. Chiang, YM, Hayman, WK: Estimates on the growth of meromorphic solutions of linear differential equations. Comment. Math. Helv. 79, 451-470 (2004)

14. Zhuang, QT, Yang, CC: The Fixed Points and Factorization Theory of Meromorphic Functions. Press in Beijing University, Beijing (1988) (in Chinese)

15. Wang, J, Yi, HX: Fixed points and hyper-order of differential polynomials generated by solutions of differential equation. Complex Var. Theory Appl. 48, 83-94 (2003)

16. Laine, I, Rieppo, J: Differential polynomials generated by linear differential equations. Complex Var. Theory Appl. 49 897-911 (2004)

17. Chen, ZX, Shon, KH: On the growth and fixed points of solutions of second order differential equation with meromorphic coefficients. Acta Math. Sin. Engl. Ser. 21(4), 753-764 (2005)

18. Liu, MS, Zhang, XM: Fixed points of meromorphic solutions of higher order linear differential equations. Ann. Acad. Sci. Fenn., Ser. A 1 Math. 31, 191-211 (2006)

19. El Farissi, A, Belaïdi, B: On oscillation theorems for differential polynomials. Electron. J. Qual. Theory Differ. Equ. 2009(22), 1-10 (2009)

20. Belaïdi, B, El Farissi, A: Growth of solutions and oscillation of differential polynomials generated by some complex linear differential equations. Hokkaido Math. J. 39(1), 127-138 (2010)

21. Chen, ZX: The relation between solution of a class of second order differential equation with functions of small growth. Chin. Ann. Math., Ser. A 27, 431-442 (2006) 
22. $\mathrm{Xu}, \mathrm{JF}, \mathrm{Yi}, \mathrm{HX}$ : Growth and fixed points of meromorphic solutions of higher-order linear differential equations. J. Korean Math. Soc. 46(4), 747-758 (2009)

23. Gross, F: On the distribution of values of meromorphic functions. Trans. Am. Math. Soc. 131(1), 199-214 (1968)

24. Gundersen, G: Estimates for the logarithmic derivative of a meromorphic function, plus similar estimates. J. Lond. Math. Soc. 37(2), 88-104 (1988)

25. Chen, ZX: Zeros of meromorphic solutions of higher order linear differential equations. Analysis 14, 425-438 (1999)

26. Chen, WJ, Xu, JF: Growth of meromorphic solutions of higher-order linear differential equations. Electron. J. Qual. Theory Differ. Equ. 1, 1-13 (2009)

27. Cao, TB, Xu, JF, Chen, ZX: On the meromorphic solutions of linear differential equations on the complex plane. J. Math. Anal. Appl. 364, 130-142 (2010)

28. Gundersen, G: Finite order solutions of second order linear differential equations. Trans. Am. Math. Soc. 305(1), 415-429 (1988)

doi:10.1186/1029-242X-2013-304

Cite this article as: $\mathrm{Xu}$ and Zhang: Some results of meromorphic solutions of second-order linear differential equations. Journal of Inequalities and Applications 2013 2013:304.

\section{Submit your manuscript to a SpringerOpen ${ }^{\circ}$ journal and benefit from:}

- Convenient online submission

Rigorous peer review

- Immediate publication on acceptance

Open access: articles freely available online

High visibility within the field

- Retaining the copyright to your article 